\title{
Implementing Primary Care Mediated Population Genetic Screening Within an Integrated Health System
}

\author{
Sean P. David, MD, SM, DPhil, Henry M. Dunnenberger, PharmD, Raabiah Ali, MPH, \\ Adam Matsil, MS, Amy A. Lemke, PhD, MS, Lavisha Singh, MPH, Anjali Zimmer, PhD, \\ and Peter J. Hulick, MD, MMSc
}

Introduction: Genetic screenings can have a large impact on enabling personalized preventive care. However, this can be limited by the primary use of medical history-based screenings in determining care. The purpose of this study was to understand the impact of DNA10K, a population-based genetic screening program mediated by primary care physicians within an integrated health system to emphasize its contribution to preventive healthcare.

Methods: Construction of the patient experience as part of DNA10K shaped the context for PCP engagement within the program. A cross-sectional analysis of patient consents, orders, tests, and results of nearly 10,000 patients within the primary care specialties of family medicine, internal medicine or obstetrics/gynecology between April 1, 2019 and January 22, 2020 was conducted.

Results: Across all specialties, a median number of 7.5 cancer and cardiovascular disease variants per PCP was found. The average age of the study population was 49.6 years. Over $8 \%$ of these patients had at least one actionable genetic risk variant and almost $2 \%$ of patients had at least one CDC Tier 1 variant. The median numbers of patients per PCP with either hereditary breast and ovarian cancer, Lynch Syndrome, or Familial Hypercholesterolemia was 1 (Interquartile Range 0-2).

Discussion: The analysis of test results and the engagement of an integrated healthcare system in the implementation of a genetic screening program suggests that it can have a large impact on population health outcomes and minimal referral burden to PCPs if identified risks can lead to preventive care. (J Am Board Fam Med 2021;34:861-865.)

Keywords: Chronic Disease, Cross-Sectional, Studies, Genetic Testing, Genomics, Integrated Health Care Systems, Primary Care Physicians, Primary Health Care

\section{Introduction}

Genetic testing for pathogenic variants that confer susceptibility for cancer and heart disease-the 2

This article was externally peer reviewed.

Submitted 23 July 2020; revised 24 July 2020; accepted 29 September 2020.

From the Pritzker School of Medicine, The University of Chicago, IL (SPD, AAL, PJH); NorthShore University HealthSystem, Evanston, IL (SPD, HMD, RA, AM, AAL, LS, PJH); Color Genomics, Inc., Burlingame, CA (AZ).

Funding: The DNA10K initiative is funded by the Transformation through Innovation Fund at NorthShore University HealthSystem. Additional funding to Dr. David from the NorthShore Auxiliary Research Award and National Institute on Minority Health and Health Disparities grant no. U54MD010724.

Conflict of interest: Dr. David acts as a scientific advisor to Genalyte, Inc. (San Diego, CA). Dr. Zimmer is employed by and owns stock in Color Genomics, Inc. (Burlingame, CA). No other disclosures were reported. most common causes of death in the United States (US) - has the potential to guide individualized disease prevention, treatment, and risk identification. Primary care physicians (PCPs) play a pivotal role in preventive services, such as applying family histories in identifying high genetic risk for disease. However, medical history-based screening can under-detect pathogenic cancer syndromes, such as hereditary breast and ovarian cancer (HBOC), Lynch

Corresponding author: Sean P. David, MD, SM, DPhil, Program Director for Translational Science, Outcomes Research Network, Vice Chair for Research, Department of Family Medicine, NorthShore University HealthSystem, Clinical Professor of Family Medicine, University of Chicago, Pritzker School of Medicine, Phone: 224-3647370 (E-mail: SDavid@northshore.org). 
syndrome, and familial hypercholesterolemia $(\mathrm{FH}),{ }^{1-3} 3$ conditions present in at least $1 \%$ of the US population considered by the Centers for Disease Control and Prevention (CDC) to be of high public health importance (CDC Tier 1 applications). The purpose of this study was to characterize the impact of PCP engagement in a population genetic screening program (DNA10K) by analyzing patient participation and test results.

\section{Methods}

DNA10K was conducted within an integrated health care system to provide genomics-guided care to more than 10,000 patients by their PCPs ( $\mathrm{n}=$ 116) in family medicine (FM), internal medicine (IM), and obstetrics/gynecology (OBGYN) across 14 clinical practice locations in northern Cook and
Lake Counties, Illinois. The context of the DNA10K implementation is described in Figure 1.

A cross-sectional analysis of consents, orders, tests, results, and actionable genetic variants of patients who were genotyped between April 1, 2019, and January 22, 2020, was conducted. Medians and interquartile (IQR) ranges of genetic test result frequencies per PCP are reported at the overall and specialty levels for cancer and cardiovascular pathogenic variants, Tier 1 pathogenic variants, ${ }^{1}$ non-Tier 1 pathogenic variants, and the percentage of actionable pharmacogenomic variants. ${ }^{4}$ Group differences for demographics and test results were examined using Kruskal-Wallis, ANOVA with posthoc Tukey's honestly significant difference (HSD) test, and Wilcoxon Rank Sum tests in Statistical Analysis System (SAS) version 9.4 (Cary, NC) with $P<.05$ being considered significant.

Figure 1. Context for Supporting PCP Engagement in Population Genetic Screening. Figure Legend: The NorthShore Center for Personalized Medicine DNA10K program consisted of multiple components. (a) patient educational video and consent via the NorthShore Connect (NSC) patient portal, (b) PCP electronic clinical decision support and electronic health record (EHR) order, (c) access to a NorthShore lab for phlebotomy, (d) Color Genomics, Inc. genetic testing using a 74 gene next-generation sequencing panel, (e) discrete reporting of results available in the EHR, (f) coordination of results with PCP health maintenance visits, (g) automated patient messaging about next steps, (h) access to genetic counseling, (i) return of results on the patient portal, and (j) clinical follow-up with PCP and specialists. The NorthShore Institutional Review Board approved this study as an exempt quality improvement evaluation. Abbreviations: PCP, primary care physician; EHR, electronic health record.

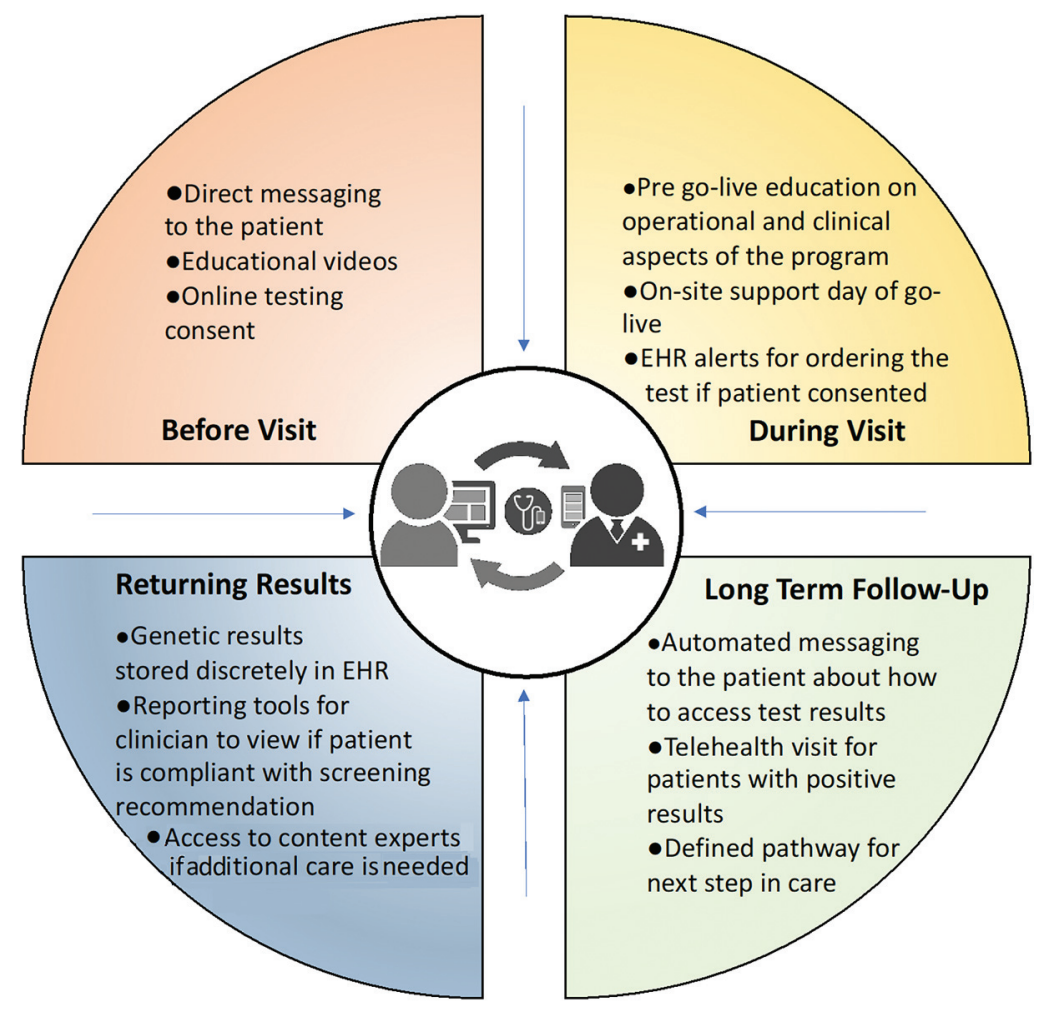




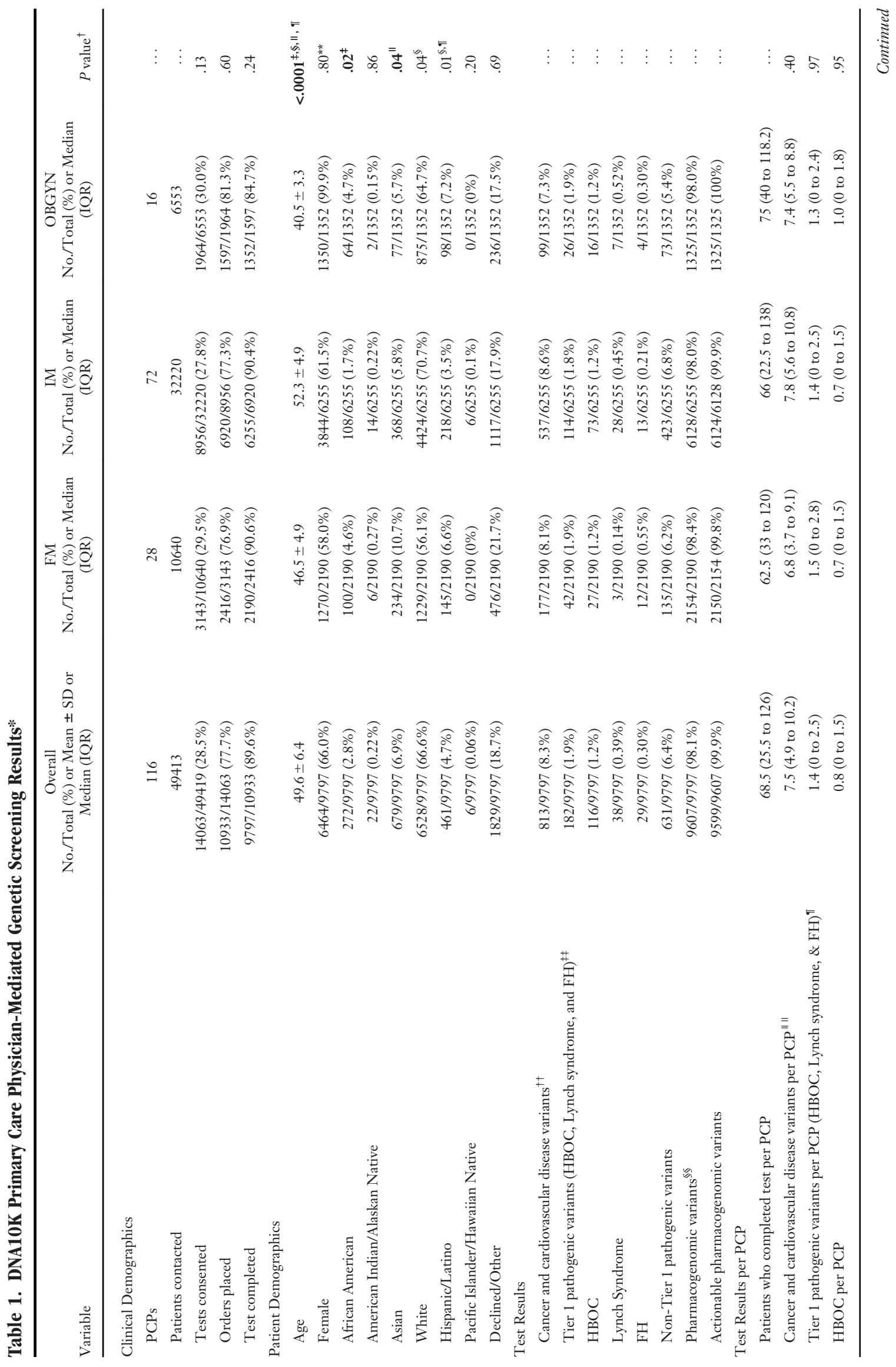




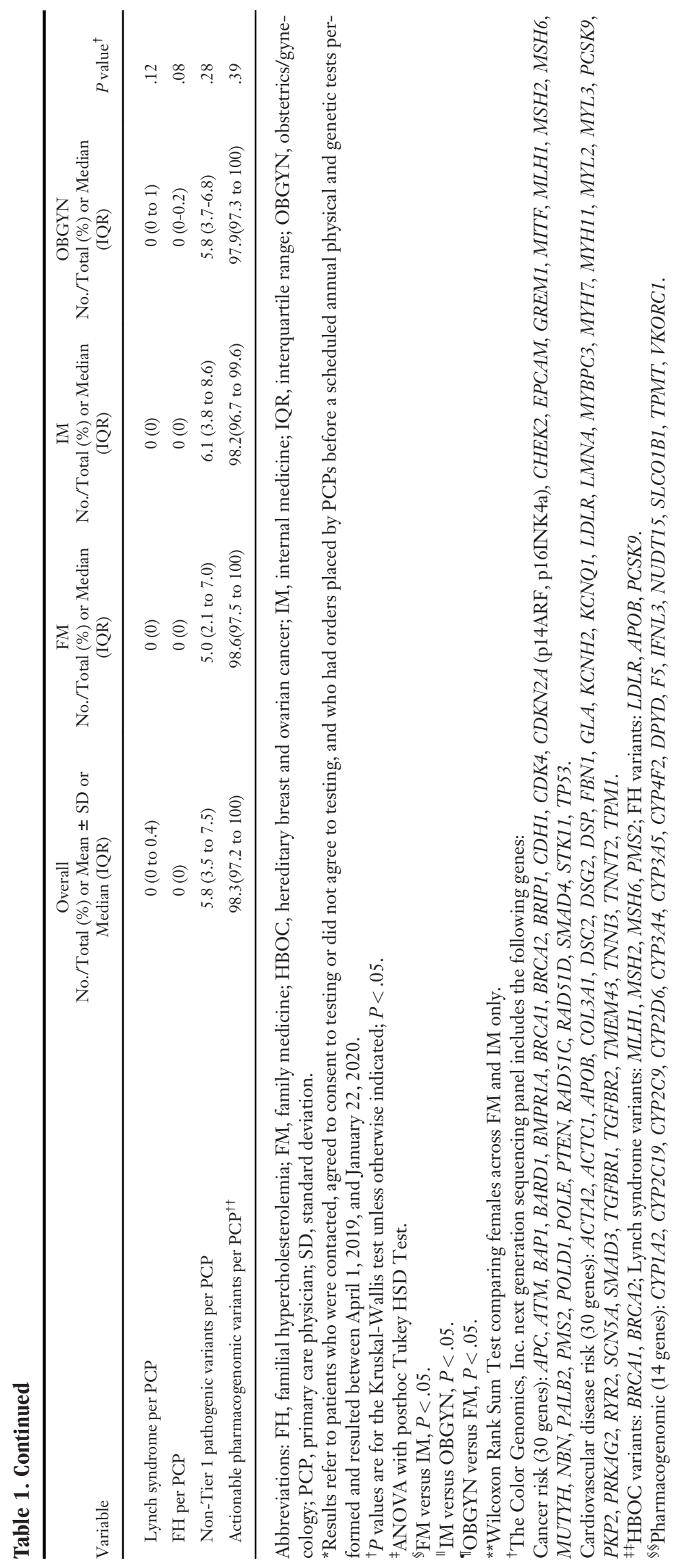




\section{Results}

Overall, 49,413 patients were contacted. Of those who consented $(\mathrm{n}=14,063), 77.7 \%(\mathrm{n}=10,933)$ had an order placed by a PCP and $89.6 \%(\mathrm{n}=$ 9,797 ) of these patients completed testing. Mean age of the participants was 49.6 years. An additional 442 patients completed testing ordered by medical geneticists and other specialists (DNA10K total $\mathrm{n}=$ 10,239). Table 1 presents stratified results by primary care specialty for DNA10K participants. A total of $813(8.3 \%)$ patients had at least 1 actionable genetic risk variant (excluding pharmacogenomics), and $182(1.9 \%)$ patients had at least 1 CDC Tier 1 variant. HBOC variants were present in 116 patients $(1.2 \%)$, Lynch syndrome in $38(0.39 \%)$ patients, and $\mathrm{FH}$ in $29(0.3 \%)$ patients. More than $99 \%$ of patients (9599 of 9607) had at least 1 actionable pharmacogenomic variant. The median number of participants per PCP ranged from 62.5 (IQR, 33 to 120) for FM, 66 (IQR, 22.5 to 138) for IM, and 75 (IQR, 40 to 118.2) for OBGYN. Median numbers of patients per PCP with at least 1 CDC Tier 1 variant or at least 1 actionable cancer or cardiovascular disease variant is 1 (IQR, 0 to 2 ) and 5 (IQR, 1 to 10$)$, respectively. No statistically significant differences were observed per specialty PCP for analyzed test results.

\section{Discussion}

In this study, the engagement and implementation of a genetic screening program in an integrated health system enabled PCPs to identify cancer and cardiovascular disease variants in more than $8 \%$ of patients and at least 1 CDC Tier 1 variant in nearly $2 \%$ of patients. Limitations included the lack of detailed patient family histories, which restricted an evaluation of its sensitivity. In addition, there was not sufficient follow-up time to assess the effects of testing on downstream screenings (eg, mammography, colonoscopy, lipid testing, genetics referrals, and medical outcomes). However, results suggest PCP-guided population-based genetic screenings could complement routine family histories to identify high genetic risks for cancer and cardiovascular disease.

We wish to acknowledge the leadership of John Revis, MD, Janardan Khandekar, MD, and Justin Brueck, MHA at NorthShore University HealthSystem (Evanston, IL), NorthShore Health Information Technology and EPIC Ambulatory Team for information technology EMR integration work, Alicia Zhou, $\mathrm{PhD}$ at Color Genomics Inc. (Burlingame, CA) for support, and EPIC Systems $^{\mathrm{TM}}$ (Verona, WI) for assistance with delivery of genomic services and EMR integration.

To see this article online, please go to: http://jabfm.org/content/ 34/4/861.full.

\section{References}

1. Murray MF, Evans JP, Khoury MJ. DNA-based population screening: potential suitability and important knowledge gaps. JAMA 2020;323:307.

2. Manickam K, Buchanan AH, Schwartz MLB, et al. Exome sequencing-based screening for BRCA1/2 expected pathogenic variants among adult biobank participants. JAMA Netw Open 2018;1:e182140.

3. Kurian AW, Bernhisel R, Larson K, et al. Prevalence of pathogenic variants in cancer susceptibility genes among women with postmenopausal breast cancer. JAMA 2020;323:995-997.

4. Relling MV, Klein TE. CPIC: Clinical pharmacogenetics implementation consortium of the pharmacogenomics research network. Clin Pharmacol Ther 2011;89:464-467. 